\title{
State-funded rehabilitation service and continuity of service in Latvia for patients with unilateral lower limb amputations: Statistical data and patients' view
}

\author{
Alisa Lubina and Daina Smite \\ Rīga Stradiņš University, Riga, Latvia
}

\begin{abstract}
Amputation is much more common than people do realize. There are no statistical data on amputations in Latvia, also any guidelines for planning, organizing and providing rehabilitation services to patients after amputation. Providing a patient-friendly and comprehensive multidisciplinary treatment is the key to successful outcomes in the case of amputation. The analysis of the current situation, including patients' view, will serve as one of the steps to improve the rehabilitation of patients with unilateral lower limb amputation in Latvia.
\end{abstract}

Key words: Latvian healthcare system, continuity of rehabilitation service, unilateral lower limb amputation.

\section{Introduction}

The main reasons for amputation are diabetes complications, vascular diseases and trauma [1]. In 2014, 2.1 million people were living with limb loss in the United States. By 2050, that rate is expected to double up [2]. It is estimated that 25-27 in 100,000 of German population will have undergone an amputation [3]. In cases of no clinical contraindication, rehabilitation should begin before amputation surgery and continue after discharge from hospital.

Rehabilitation activities are divided into different phases, in different guidelines are given different phase names, but most phases are: preoperative phase, operation phase, postoperative phase, rehabilitation phase, post-prosthetic rehabilitation and lifelong care. All patients, including those who are not prescribed prosthetics, should have the opportunity to participate in a rehabilitation program in accordance with the policies and procedures of the treating institution [4]. It is important that the rehabilitation process is successive and involves a multidisciplinary team. It is important that the professionals involved in it, work as a team and that the patient with his or her relatives is also seen as part of the team. Rehabilitation should be patient-centred, responsive to their needs, considering the physical and emotional difficulties they face [5]. Prosthetic services play a crucial role in the rehabilitation process for patients with lower limb amputations. However, literature review data shows that prosthetic rejection varies from 0 to 50 percent between prosthetic patients. The main indicators for not using the prosthesis are: low level of satisfaction of functional needs, weight of prosthesis, discomfort from the socket [6].

The procedure for receiving state-funded rehabilitation services in Latvia is determined in accordance with Regulations No. 555 of 28 August 2018 "Procedure for Financing and Organizing Health Care" (Regulations No. 555). According to Regulations, the purpose of medical rehabilitation services is to provide service, reduce limitations and to provide 
assessment and reduction of the risk of complications for people with certain functional limitations. A doctor of physical and rehabilitation medicine performs selection of patients for medical rehabilitation services. Medical rehabilitation services with referral from rehabilitation medical doctor and in accordance with the elaborated rehabilitation plan, a person may receive in an outpatient medical institution, including day hospital or in hospital (inpatient rehabilitation).

The aim of the study was to analyze state-funded rehabilitation services and its continuity in Latvia for patients with unilateral lower limb amputation based on statistical data and patients' view.

\section{Methods}

Research design is a case study. As a real-life context Latvian healthcare system was identified and as a phenomenon - state-funded rehabilitation service and continuity of service for patients with unilateral lower limb amputation. Phenomenon was divided into two parts (1) state-funded rehabilitation service and continuity of it and (2) state-funded rehabilitation service in Latvian healthcare system. The first part includes statistical data and patient's opinion on in-patient rehabilitation services (duration, place of delivery, characteristics of the recipient, patient's view (satisfaction). It also includes outpatient rehabilitation services (type and form, volume, time of receipt, character of the recipient, patient's view (satisfaction); continuity of rehabilitation after amputation surgery (time from amputation hospitalization episode to rehabilitation service, service frequency - time and type, outpatient service and inpatient service sequence. Patients' view of service continuity means - whether they have successive sequences and limiting factors). Data sources were Medical information system (MIS) data (quantitative data) and patient semi-structured interviews (qualitative data). Second part - state-funded rehabilitation service in Latvian healthcare system - includes statistical analysis: the general characteristics of the patients (sex, age, level and cause of amputation, hospital) and their treatment after amputation (all healthcare services) - hospital episodes, outpatient service and manipulations. Data sources were MIS data (quantitative data).

Participants in the study were people, who during the period between 01.02.2016 and 31.05.2016 (four months) underwent unilateral lower limb amputation - total number - 234 unique patients: 148 men and 86 women (33 died during hospital stay).

For qualitative data research, a group of 5 patients (P1, P2, P3, P4, P5) were enrolled intentionally based on selection criteria - included in the study patients have received statefunded inpatient rehabilitation service in National Rehabilitation Centre "Vaivari" (NRC Vaivari). Considering the small number of participants, who were successful in applying the selection criteria, extra patients were selected (similar to the selection criteria) - extra 3 patients after unilateral lower limb amputation (amputation time 2016 - beginning of 2017) and who received state paid inpatient rehabilitation services in NRC Vaivari at the beginning of 2018 .

Data on the state-paid inpatient and outpatient health care and rehabilitation services provided to persons who had unilateral lower limb amputation between 01.02.2016 and 31.05.2016 was selected from MIS.

Data on all services after amputation and until 01.10.2017 was collected and analyzed (i.e., 17 (20) months after amputation surgery).

Stage 1 - Data selection from inpatient service database on amputation hospitalization to create and characterize study participants (analysis units). Data on hospitalization was selected for the period starting on 01.02.2016 until 31.05.2016 - specific NOMESCO surgical 
Table 1. Description of inpatient rehabilitation service and recipients.

\begin{tabular}{|c|c|c|c|}
\hline Hospital & Patients & Duration & Description \\
\hline $\begin{array}{l}\text { NRC } \\
\text { "Vaivari" }\end{array}$ & 6 & $\begin{array}{l}13(n=4) \\
14(n=2) \\
\text { median 13 }\end{array}$ & $\begin{array}{l}\text { Level: transfemoral }(n=5) \text {; hip exarticulation }(n=1) \\
\text { Cause: circulatory disorders }(n=3) \text {; muscle and } \\
\text { skeletal system damage }(n=2) \text {; skin and } \\
\text { subcutaneous damages }(n=1) \\
\text { Sex: female } n=4 / \text { male } n=2 \\
\text { Age }(\text { years }): n=1(15-19) / n=1(50-54) / n=2 \\
(70-74) / n=1(75-79 / n=1(85-89)\end{array}$ \\
\hline $\begin{array}{l}\text { Liepaja } \\
\text { regional } \\
\text { hospital }\end{array}$ & 1 & 4 & $\begin{array}{l}\text { Metatarsophalangeal surgery } \\
\text { circulatory disorders } \\
\text { Male, age: } 60-64 \text { y. }\end{array}$ \\
\hline
\end{tabular}

manipulation classifier (NCSP) manipulations were performed (excluding performed ZXA10 (bilateral) manipulation).

Stage 2 - Data selection from inpatient service database for inpatient patient care after amputation. Data on hospitalization of amputation patients after amputation and until 01.10.2017 was selected. Totally 257 hospitalizations were selected.

Stage 3 - Data selection from outpatient service database for outpatient patient care after amputation. Data on outpatient services for amputation patients after amputation and until 01.10.2017. In addition, outpatient service manipulations were selected and summarized: manipulation code, name and number.

The first part of the phenomenon was described by both quantitative and qualitative data, so a sequential, combined approach was used in the data analysis, i.e. the initial quantitative data were processed and analyzed using mathematical statistical methods (Microsoft excel and StatPlus version 6.2.30). Frequency analysis, median and median absolute deviation, minimum and maximum value, sample mean. For visual analysis, graphic images were used, considering each random participant. In turn, qualitative data from interview transcripts were initially analyzed using qualitative content analysis. Thematic analysis was used for the processing and analysis of qualitative data (patient interview transcripts). Transcript analysis included six steps: accessing data, primary coding, topic queries in primary codes, topic overview, topic definition and naming, analysis overview. In the second stage of the analysis, the data were combined, using qualitative data as a complement to the quantitative indicators, creating an individual-oriented analysis model.

At the end of the analysis of each part of the phenomenon, regularities were searched and their explanation was integrated with the theoretical findings (inductive analysis strategy (Yin (1984a, 2014b)), forming hypotheses. The first phenomenon was analyzed in context with the second phenomenon, highlighting regularities, integrating theoretical concepts and hypotheses.

\section{Results}

Eleven out of 201 (5.5\%) patients received rehabilitation services (after acute hospitalization, due to amputation, discharged from hospital). Seven out of these 11 patients received inpatient rehabilitation. Table 1 presents the characteristics of the recipients and description of inpatient rehabilitation services.

Six patients received outpatient rehabilitation services after amputation. Characteristics of the recipients of outpatient rehabilitation services and services are presented in Table 2. 
Table 2. Description of outpatient rehabilitation service and recipients.

\begin{tabular}{|c|c|c|c|c|c|c|}
\hline Specialist & $\begin{array}{l}\text { Number } \\
\text { of } \\
\text { episodes }\end{array}$ & Patients & $\begin{array}{l}\text { Duration of } \\
\text { episodes } \\
\mathrm{V}(\mathrm{M}(\mathrm{MAD})) \text {; } \\
\mathrm{min} / \max \end{array}$ & $\begin{array}{l}\text { Diagnosis } \\
\text { (code) }\end{array}$ & $\begin{array}{l}\text { Referral } \\
\text { from }\end{array}$ & Service \\
\hline P54 & 11 & 9 & $\begin{array}{l}8 \text { consultations (1) } \\
\text { Multiprofessional } \\
\text { team } \\
10(6(0)) 5 / 19\end{array}$ & $\begin{array}{l}\text { I69; I70.2; } \\
\text { I83; T93.6; } \\
\text { T93.8; } \\
\text { Z48.8; } \\
\text { C49.8 } \\
\text { Z96.6; } \\
\text { I70.2; T93.8 }\end{array}$ & $\begin{array}{l}\text { P02 }(n=7) \\
\text { P03 }(n=1) \\
\text { P54 }\end{array}$ & $\begin{array}{l}n=7 \\
\text { outpatient } \\
n=1 \\
\text { home visit }\end{array}$ \\
\hline T01 & 2 & 2 & $9(9(3)) 6 / 12$ & I70.2; C48.2 & P54 & Outpatient \\
\hline T02 & 3 & 2 & $\begin{array}{l}10.3(10(1)) \\
9 / 12\end{array}$ & I69; I70.2 & P54 & Home care \\
\hline
\end{tabular}

P54 - Rehabilitation doctor, P02 - Internal medicine doctor, P03 - surgeon, T01 - Physiotherapist, T02 - Occupational therapist.

Table 3. Continuity of rehabilitation service.

\begin{tabular}{|l|l|}
\hline Rehabilitation services & Days since amputation V (M (MAD)); min/max \\
\hline Inpatient rehabilitation & $230.1(\mathbf{1 7 5}(1)) 143 / 377$ \\
\hline Rehabilitation doctor's consultation & $158.9(\mathbf{1 3 0}(113)) 33 / 348$ \\
\hline $\begin{array}{l}\text { Physiotherapist, Occupational therapist or } \\
\text { multi-professional rehabilitation service }\end{array}$ & $103.6(\mathbf{8 5}(124)) 43 / 172$ \\
\hline
\end{tabular}

$\mathrm{V}$ - average value, $\mathrm{M}$ - median, MAD - median absolute deviation, min/max - minimum/maximal value, * the number of days since the discharge from the hospital after the amputation operation.

Analyzing continuity of rehabilitation services, time (number of days) between amputation operation and received rehabilitation services was calculated (Table 3).

After inpatient rehabilitation, which lasted 40 days after discharge from inpatient rehabilitation, only one patient received outpatient rehabilitation in a day care service (5 days).

More often amputations were performed for males aged 65-69 $(n=34)$ and females aged $70-74(n=14)$ and $80-84$ years $(n=14)$, while in age groups 5-9 years and $15-19$ years only 2 patients were selected $(n=1, n=1)$. See detailed information in Fig. 1.

After an amputation operation $85.90 \%(n=201)$ patients were successfully discharged from hospital, while $14.10 \%(n=33)$ of patients died in hospital. The number of bed days in a hospital after an amputation operation: on average $19.1 \pm 16.1$ days (median -16 , MAD $-8, \min / \max -1 / 100)$ in day care.

$67.92 \%(n=163)$ of amputation was caused by diseases of the circulatory system, $12.08 \%(n=29)$ was caused by injuries, poisoning and other effects of frost (frostbite), less often - malignant tumours $-1.67 \%(n=4)$ and 1 patient, or $0.42 \%$, were reported as gangrene, not classified elsewhere.

In $56.23 \%$ of cases $(n=158)$, patients had transfemoral amputation, the least frequent patients had hip exarticulation $-1.07 \%(n=3)$.

Overall, in the period of 1 day to 20 months after discharge from hospital after amputation, 257 hospitalizations were selected with 123 unique patients. In 73.17\% ( $n=90)$ cases, 


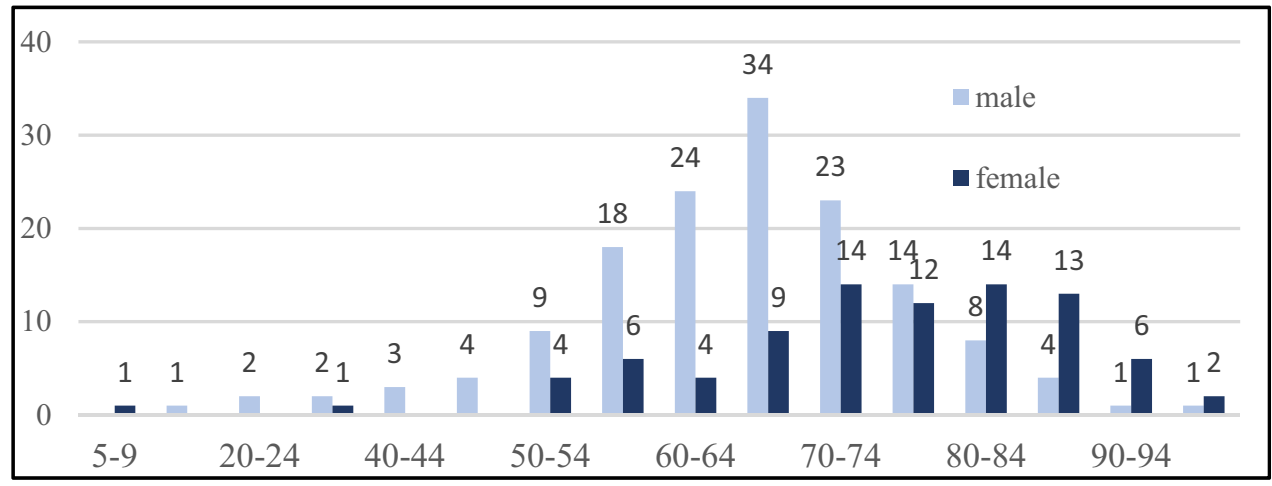

Fig. 1. Distribution of patients with amputation by age and gender.

patients were successfully discharged from hospital after inpatient treatment, while in $26.83 \%$ $(n=33)$ patients died in hospital.

Outpatient services were characterized by 2,462 outpatient episodes with 185 unique patients. Most often patients $(n=160)$ had attended a family doctor -914 care episodes (66.42\% of the total number of consultations), with an average for one patient 5.7 times. Rehabilitation doctor provided $0.6 \%$ of all outpatient consultations.

More frequent outpatient manipulations were selected from MIS data. Most frequent manipulation - laboratory tests, in total 2576 manipulations.

The number of rehabilitation manipulations was 55 ( $0.51 \%$ of the total) with an average of 2.9 in one care episode and with a maximum of 10 times in one care episode.

Interview transcripts were analyzed by selecting meaningful (illustrative) phrases and creating primary codes with the help of computer software NVIVO. As a result, the patients' view of state-paid rehabilitation services and their succession was obtained on 6 topics. They included the content and satisfaction of rehabilitation service in the acute hospital, the content and satisfaction of rehabilitation service in inpatient rehabilitation, the content and satisfaction of rehabilitation service in the outpatient sector, information about rehabilitation services, barriers to receiving rehabilitation services and contributing factors for receiving rehabilitation services.

The topic "the content and satisfaction of rehabilitation service in the acute hospital" was characterized by 5 sub-themes. One of them was mobility and mobility training / training with a physiotherapist (characterized by illustrative quotes from 4 interviews). Others were lack of rehabilitation measures (characterized by a quote from the P4 interview), lack of psychological help (described by one quote from the P2 interview), satisfaction with physiotherapy services (reflected in P1 and P3 interviews), and dissatisfaction with the quality of physiotherapy service (characterized by one illustrative quote from P2 interview).

The topic "Content and satisfaction of rehabilitation service in inpatient rehabilitation" was characterized by 4 sub-themes. They included - received various treatment technologies (illustrated by quotes from all interviews), inconsistency in intensity and / or volume (characterized by illustrative quotes from interviews P2, P3 and P4.), rehabilitation improved mobility (reflected in interviews P1, P3 and P5), and satisfaction with the overall quality of service and staff attitude (characterized by illustrative quotes from all interviews).

The topic "Content and satisfaction of rehabilitation service in the outpatient sector" was characterized by 2 sub-themes. They were - received physiotherapy services in the form of a day hospital after the acute phase (described by illustrative quotes from interviews P1, P2 and 
P3), and transport and mobility barriers for outpatient services (characterized by illustrative quotations from interviews $\mathrm{P} 1, \mathrm{P} 2$ and $\mathrm{P} 3$ ).

There were 3 sub-themes for the topic "Information about rehabilitation services": Lack of information from health care professionals (acute inpatient or GP) (reflected in all interviews), the Internet as a source of information (described by quotations from interviews P1 and P2), and family support for information (reflected in quotes from interviews P3, P4 and P5).

The topic "Barriers to receiving rehabilitation services" was characterized by 6 subtopics. They included: 1) a lack of information at all stages of treatment (illustrative quotes from all interviews); 2) a lack of knowledge of the attending physician about rehabilitation (quotes from P5 interview); 3) transport and mobility barriers to outpatient services (quotations from interviews P1, P2 and P3); 4) environmental barriers (characterized by quotations from interviews $\mathrm{P} 1, \mathrm{P} 2$ and $\mathrm{P} 4$ ); 5) personal factors as a barrier (quotes from interviews P1, P2 and P5); and 6) waiting queues (reflected in quotes from interviews P1 and P5).

The topic "Contributing factors for receiving rehabilitation services" was characterized by 2 subtopics: financial opportunities for receiving paid services (illustrative quotes from interviews P4 and P5), and support from relatives (characterized by illustrative quotes from interviews P1, P3, P4 and P5).

\section{Discussion}

Selected research design - case study allowed us to analyze both phenomena in Latvian health care system. Rehabilitation after amputation is a complex process where a patient receives professional help and support after the loss of the limb. The purpose of rehabilitation is to achieve patient's independence in all areas of life and without functional limitations [7].

The results of the study showed that only $5.47 \%$ of the total number of patients, discharged from hospital in the next 17-20 months, received any kind of state-funded medical rehabilitation service. Patients after the lower limb amputations do not actually receive state-funded medical rehabilitation. This argument could assume that some patients do not have adequate rehabilitation potential, or patients are reluctant to engage in rehabilitation activities. However, guidelines for rehabilitation programs for patients after amputations emphasize that rehabilitation should be patient-oriented, tailored to the patient's changing needs and individual abilities, also comprehensive multi-professional team approach is provided $[8,9]$.

Considering that mostly transfemoral amputations were performed, patients had problems with mobility and day-to-day activities, requiring the need for technical aids and other adaptations to the new situation, raising the need for rehabilitation. Moreover, if we assume that in an acute hospital early after amputation (average number of bed days 19.1 \pm 16.1 days), unstructured rehabilitation services (e.g. separate physiotherapist and/or occupational therapist activities) were implemented, then without any planned and structured rehabilitation measures, their usefulness is insignificant. Unfortunately, the patients' view of rehabilitation service in the early hospital shows their dissatisfaction with its quality. Patients complained: "It came five times, of course, came in half an hour, fast, fast, let's say it was very poor..." or complete absence ("cut off and that was it, nothing more left (...), nothing more to do, so I slept all day (...)"). As well, two important topics pop up - a lack of information about rehabilitation possibilities, what possibilities exist at all that combine with psychological support.

It has been proven that depression, alcohol addiction or post-traumatic stress syndrome are often observed in patients after amputations. The guidelines found in the literature 
review emphasize that during the entire rehabilitation process the patient, patient's family members and caregivers need long-term psychological support. Unfortunately, in the current state-funded rehabilitation services, offered for patients after amputations in Latvia, there is practically no psychological help. Thus, when designing a structured rehabilitation program for patients after amputations, we should think about how to include psychologist / psychiatrist / psychotherapist services on early stages, as well as train every member of a multidisciplinary team to provide quality emotional support to patients after losing their limb. It is equally important to remember the patient's relatives both as an indispensable resource for supporting (also highlighted by the results of the interviews) and as a target group for receiving support, also need to adapt to the new situation and protect themselves from exhaustion in the informal care process.

According to the analysis of interviews, patients underline the lack of information as the main barrier and deficiency at all stages of treatment - there was a lack of information both from hospital staff, how to proceed, and from the family doctor. All interviewed patients confirmed that information on rehabilitation services were searched on the Internet, or their relatives searched for information. The issue of who needs to be the main provider of information on rehabilitation possibilities - the treating doctor (i.e., the surgeon), rehabilitation doctor, the functional specialist, the family doctor, the nurse, or anyone involved in the care (treatment), becomes more topical. Based on good practice models, in the absence of clinical contraindications, the rehabilitation program should start before the amputation operation (in cases where amputation is planned) [10]. Thus the patient should receive information about further rehabilitation and its possibilities before the amputation operation, or immediately afterwards. As with other scheduled operations (e.g. joint endoprosthesis or oncology patient care), patients receive information materials (printed or electronic) as early as possible, as well as the development of an individual, patient-coordinated rehabilitation plan creation is required.

Analysis of the results of medical rehabilitation services, received by patients after unilateral lower limb amputation from 17 to 20 months after discharge from an acute hospital, showed that only 7 patients have received inpatient rehabilitation service. In turn, 10 patients have received one of the outpatient rehabilitation services, including day care, (6 of them were patients who had also received inpatient rehabilitation).

Inpatient rehabilitation services were received in NRC Vaivari (6 out of 7 patients) for 13 or 14 days. Literature data suggest that the duration of inpatient rehabilitation should depend on the purpose of the rehabilitation and the condition of the patient, so each patient may have a different number of bed days [11]. This research did not include an in-depth analysis of the contents of the rehabilitation service, but patients' views on inpatient rehabilitation show that all patients have received similar services and services amount - physiotherapy classes, pool, pearl baths, massage and physical procedures. All patients were satisfied with the quality of the overall service and staff's attitude, but were not satisfied with the intensity of the service, indicating that it could be more frequent.

Outpatient rehabilitation services included both Rehabilitation doctor's consultation, training with functional specialist and multi-professional rehabilitation in day-care. Unfortunately, it is worth noting that the statistical results show that only $0.65 \%$ or 16 (out of 2462) outpatient care episodes related to rehabilitation services. Analysis of the patient's view on the content and satisfaction of rehabilitation measures showed that the availability of outpatient services is hampered by restrictions on transport and mobility. As an alternative to outpatient rehabilitation services, there could be services at patient's home. At present, patients after amputation are not able to receive state-funded medical rehabilitation at home (only patients with diagnosis I63 or I70.2). Considering environmental barriers, infrastructure 
and localization of medical institutions, medical rehabilitation services at home could be the optimal solution for patients after amputation. Of course, this will raise the need for professionals (physiotherapists and physiotherapists' assistants, occupational therapists and occupational therapists' assistants) in the regions and review the issue of the appropriate cost.

Analyzing data on service continuity in Latvia, we concluded that rehabilitation services were not provided continuously 85 days to outpatient service (in case of inpatient consultation of Rehabilitation doctor in hospital), all services were delayed. Only one patient received outpatient rehabilitation after inpatient rehabilitation, although patients did not reach the goal of rehabilitation (independent movement with non-assistive prosthesis), opinion proved based on the results of patient interviews.

The results of the research show that patients after inpatient rehabilitation are no longer provided with rehabilitation services. The follow-up phase (rehabilitation after postprosthetic rehabilitation and lifelong care) in Latvia is not being implemented and the needs of the patient, which may change over time, are not considered. Of course, it should be considered that the availability of outpatient services was limited by the availability of already mentioned transport and mobility and by personal factors.

The chosen research methodology allowed us to achieve the set goal and tasks. Collaboration with the NHS helped to produce results. However, it should be considered that the MIS is a system for monitoring financial resources rather than a patient-specific database. Based on MIS data, state-funded rehabilitation services and continuity of service were analyzed. Interviews with patients who have received state-funded rehabilitation services made it possible to find out the patients' views on rehabilitation services, continuity and limiting factors.

It should be noted that there have been no studies in Latvia on rehabilitation services and continuity of it, as well as patients' views. The results of the study show that it would be useful to include the analysis of the content of services (in-patient services) as well as the description of functional assessment and goals and their achievement into next research.

\section{Conclusion}

State-funded medical rehabilitation services, including outpatient and inpatient ones, received by patients $(5.5 \%)$ with unilateral lower extremity amputation (mostly transtibial amputations due to peripheral circulatory diseases) within 17 to 20 months after discharge from the acute hospital post unilateral lower limb amputation, were not provided continuously because the service was late initiated, and did not continue after post-prosthetic rehabilitation. This finding should be seen in the context of the unmet need for information on rehabilitation opportunities expressed by the patient and the unstructured rehabilitation services received during the acute post-operative period and the lack of psychological assistance.

Analyzing state-funded medical rehabilitation services in the context of state-funded health care services for patients with unilateral lower limb amputation, one can see that there are ways to integrate long-term or less specific rehabilitation services into the overall patient care plan (e.g., care hospital or integrating rehabilitation service into care beds profile, nursing care resources, and medical assistants' home visits).

Patients' satisfaction with the content and quality of state-funded rehabilitation service at different stages of treatment was different, but there were common barriers to receiving services - lack of information, inaccessibility of outpatient services due to transport and mobility barriers, waiting lists for in-patient services, as well as personal factors. This finding presents possible short-term interventions (e.g. development of information material, introduction of a medical rehabilitation service for a particular patient group, selection of 
patients and prioritization of rehabilitation) in order to reduce the barriers for receiving existing rehabilitation services.

\section{References}

[1] P.L. Chalya, J.B. Mabula, R.M. Dass, I.H. Ngayomela, A.B. Chandika, N. Mbelenge, J.M. Gilyoma, J. Orthop. Surg. Res. 7(18), (2013)

[2] Department of Veterans Affairs/Department of Defense. Clinical practice guideline for rehabilitation of lower limb amputation (2007). Available: http://www . healthquality.va.gov/guidelines/rehab/amp/amp_v652.pdf

[3] K. Kröger, C. Berg, F. Santosa, N. Malyar, H. Reinecke, Deutsches Arzteblatt Int. 114(7), 130-136 (2017)

[4] New South Wales Health, Amputee Care Standarts in New South Wales. Reviewed at 2016. Available: http://www.health.nsw.gov.au/policies/pd/2008/ pdf/PD2008_015.pdf

[5] Statewide Rehabilitation Clinical Network. Model of Amputee Rehabilitation in South Australia (2012). Available: http://www.sahealth.sa.gov.au/wps/wcm/ connect/7b1770804b32fa9e8708afe79043faf0/Amputee+Rehabilitation+ Model+of+Care $\cdot$ pdf ?MOD=A JPERES

[6] S. Carey, D. Lura, M. Highsmith, J. Rehabilit. Res. Dev. 52(3), 247-262 (2015)

[7] I. Kovac, N. Kauzlaric, O. Živkovic, V. Mužic, M. Abramovic, Z. Vauletic, T. Vukic, N. Ištvanovic, B. Livakovic, Period. Biolog. 17(1), 147-159 (2015)

[8] M.A. Pet, S.D. Morrison, J.S. Mack, E.D. Sears, T. Wright, A.D. Lussiez, K.R. Means, J.P. Higgins, J.H. Ko, P.S. Cederna, T.A. Kung, Injury 47(12), 2783-2788 (2016)

[9] Carf International, Medical rehabilitation Program Descriptions (2018). Available: http://www . carf .org/Programs/Medical/

[10] C.P.F. Pasquina, A.J. Carvalho, T.P. Sheehan, Amer. Med. Asso. J. Ethics 17(6), 535$546(2015)$

[11] A. Kayssi, S. Dilkas, L.D. Dance, C. Mestral, T.L. Forbes, G. Roche-Nagle, PM\&R 9(5), 494-501 (2017) 